\author{
LVOV MATHEMATICAL SCHOOL \\ IN THE PERIOD 1915-45 AS SEEN TODAY \\ BANACH CENTER PUBLICATIONS, VOLUME 87 \\ INSTITUTE OF MATHEMATICS \\ POLISH ACADEMY OF SCIENCES \\ WARSZAWA 2009
}

\title{
FUNCTIONAL ANALYSIS IN LVIV AFTER 1945
}

\author{
HALYNA I. CHUYKO \\ Faculty of Mechanics and Mathematics \\ Ivan Franko National University \\ UA-79000 Lviv, Ukraine \\ E-mail:galina_chuiko@yahoo.com
}

World War II destroyed the Lviv Mathematical School: Stefan Banach died in 1945 of an illness, some of Lviv mathematicians were killed, some others disappeared. Those who survived left Lviv for Poland and other countries.

But Lviv did not become a remote mathematical province. Such mathematicians as B. Gnedenko, Al. Kovan'ko, Iv. Sokolov, later L. Volkovysky, Ya. Lopatinsky started to work at the University of Lviv. Their scientific interests were quite different: the theory of probabilities, the theory of almost periodic functions, the constructive theory of functions, complex analysis, differential equations. But they used the concepts and methods of functional analysis in their research and the special courses. The seminar on the spectral theory in Hilbert space headed by Gnedenko played a central role in forming the direction of forthcoming investigations in Lviv.

It is impossible to give a full and precise description of all results obtained in the post-war period in Lviv. We shall mention only some of them.

The most prominent figure in the Lviv mathematical life in the afterwar period was Ya. Lopatinsky. Although he dealt with differential equations, some of his results are in foundations of the modern theory of boundary problems, in particular, the well-known Lopatinsky-Shapiro condition which is sufficient to reduce an elliptic boundary problem to a system of regular integral equations. In connection with quantum field theory O. Parasyuk obtained significant results on the problem of defining the product of distributions. Academician Bogolyubov, the Nobel laureate, appreciated these results very much. A. Volpert developed an effective algorithm of calculating the index of an elliptic problem before the Atiyah-Singer index theorem appeared.

A new stage in the development of functional analysis in Lviv is connected with the name of Władysław Lance $(=\mathrm{V}$. Lyantse). As a student of the University of Lviv in

2000 Mathematics Subject Classification: Primary 01A72; Secondary 46-03.

The paper is in final form and no version of it will be published elsewhere. 
1940-1941 W. Lance took the courses of prominent mathematicians such as W. Orlicz, J. Schauder, and S. Mazur. ${ }^{1}$ He took part in the Banach seminar. Prof. Lance said: Even now I cannot believe that Professor Banach shook my hand. I was just a student. Although Banach understood the power of his talent, his way of behaving with young mathematicians was quite simple.

As a second year student W. Lance obtained his first scientific result. He proved a theorem answering the Szpilrajn problem. He was very much surprised to see the reference to this theorem in the American edition of "The Scottish Book" many years later, after the war. He was so astonished because the theorem was not published before the war and Lance's fate was unknown to the publishers of "The Scottish Book".

W. Lance graduated from the University of Lviv after the war and some years later he obtained his Ph.D. under the supervision of Yaroslav Lopatinsky. So his first love in mathematics were differential equations. Yet, he successfully used the methods of the theory of operators in his work. In 1964 W. Lance defended his Doctor's degree thesis in the Moscow State University. The main results of his thesis concern the theory of generalized spectral operators and its applications to the spectral analysis of the SturmLiouville operator with complex potential on a semiaxis.

Investigating the correctness of the mixed problem for a strongly parabolic system W. Lance found a condition under which a linear operator is the generator of a strongly continuous contracting semigroup in a Hilbert space.

During the second half of the 20th century operator theory developed from a subdomain of functional analysis to a fast increasing domain by itself. The spectral theory of linear operators has been recognized from the early beginning as the most important due to its applications to ordinary and partial differential equations. The monumental three volume monograph of N. Dunford and J. T. Schwartz "Linear Operators" was meant to be a panorama of the domain and an ultimate source of reference, as well.

In this respect, the class of so-called spectral operators in the Dunford sense was considered for some time as the peak of the spectral theory of linear operators. However, it was soon admitted that beyond this class there existed a vast field of investigations. One of the new directions of investigation based on spectral subspaces or spectral functional calculi was developed in Romania by C. Foiaş and I. Colojoara. Another direction started by M. Naǐmark was worked out in Lviv by W. Lance and his school. M. Naǐmark showed (first for the non-selfadjoint Sturm-Liouville operator on a semiaxis) that in the continuous spectrum of this operator there exist some specific points, called spectral singularities. Thanks to them the spectral measure of the operator is unbounded in norm. An isolated spectral singularity is a pole of the resolvent under its analytic extension through the continuous spectrum. So it is an obstacle to build the spectral expansion. W. Lance showed that the operator with spectral singularities has a spectral expansion. This expansion can be represented by a regularized divergent integral. For an operator with a

\footnotetext{
${ }^{1}$ Among the students of those courses were Mark Wiszik (= Vishik) and Abraham Strauss. Both of them became well-known mathematicians. M. Wiszik many years worked as professor of the State University of Moscow. A. Strauss (1920-1999) was a professor of Ulyanovsk Teachers' Training Institute. W. Lance and M. Wiszik celebrated their 85th birthday in 2005.
} 
finite number of spectral singularities the Fourier transformation can be built, but in this case the transformation has a finite-dimensional kernel. However, it is useful to resolve evolution problems. The same results have been obtained for a non-selfadjoint difference operator and for smooth non-selfadjoint perturbations of the operator of multiplication by an independent variable (non-selfadjoint Friedrichs' model).

Yaroslav Mykytyuk proved some results on non-selfadjoint elliptic operators with spectral singularities. Moreover, he found conditions under which the set of spectral singularities is bounded, closed and is of linear Lebesgue measure zero. At present Ya. Mykytyuk fruitfully cooperates with Rostyslav Hryniv. They investigate the Sturm-Liouville operator with singular potential and inverse spectral problems. These are important due to various applications to problems of mathematical physics. They have found conditions under which the point spectrum of the Sturm-Liouville operator on the whole axis with singular periodic potential is empty. Moreover, they investigated the problem of reconstruction of potential from three spectra, from two spectra, and from a spectrum and the sequence of normalizing factors.

Applying a soliton technique Igor Suroid obtained conditions of spectrality (in the Dunford-Bade sense) for the Schrödinger and Dirac operators. Eugen Cheremnykh investigated the Friedrichs model on a compact interval. He studied the properties of pseudoresolvents, their points of branching at the boundary of spectrum, the existence of extension out of space. He built spectral expansions for some non-selfadjoint difference and differential operators.

For a deeper understanding of spectral singularities W. Lance, V. Blashchak, I. Syroid, and Eu. Cheremnykh investigated the non-selfadjoint inverse problem of spectral scattering theory. The Marchenko method was applied but it was necessary to change the scattering data. Some difficulties in the proof of unique solvability of the Marchenko equation have been overcome by reducing that equation to an integral equation of the Fredholm type.

In a neighbourhood of a spectral singularity the spectral projectors are unbounded in norm. This was the starting point for generalizations of the notion of spectral measure and spectral operator (W. Lance, H. Chuyko). First, a generalized spectral measure (g.s.m.) is defined on Borel sets which are at a positive distance from the spectral singularities. The g.s.m. admits extensions, among them the maximal one. Using the technique of equipped spaces the maximal g.s.m. is extended to the algebra of all Borel sets of the complex plane (its values may be unbounded projectors). It has been proved that this extended g.s.m. is similar to a resolution of identity of a normal operator, however, the similarity is established by an unbounded, closed, densely defined operator. For the operators which commute with g.s.m. the canonical representations have been obtained.

Another direction of investigation in Lviv is the theory of "related" operators (G. Keselman, V. Kolisnyk, W. Lance, O. Storozh, M. Fedyk). We use the name "related" for the class of operators which are obtained from one another by means of finite-dimensional extensions and restrictions.

Here we enumerate only some facts of the spectral theory of related operators: the general form of an operator related to a given one; formulae connecting the resolvents 
of related operators; asymptotic formulae for eigenvalues and eigenelements; spectral expansions for some operators related to the differential operators; sufficient conditions for selfadjointness of such operators.

Later the theory has been extended to some types of infinite-dimensional (in particular, L-compact) perturbations with change of domain. Applications to differentialboundary operators with integral boundary conditions have been found. Moreover, Oleg Storozh obtained conditions of maximal dissipativeness and maximal accretiveness of related operators. He has described also the maximal accretive extensions of a coercive operator with finite defect index. It is of interest to generalize this result to arbitrary non-negative operators (the Phillips problem, 1957). Some problems of the theory of perturbations with changing the action of operator and its domain have been investigated by Olga Mylyo, Orest Shuvar, and Hanna Pipa.

Mikhail Fedyk proved some results on operators related to sesquilinear forms. Such operators preserve the properties of a given form, namely, coerciveness or sectorialness. In the case of a differential operator this class includes operators with various singular or integro-differential (non-local) boundary conditions and singular potential, for example, the operators of many-point problems.

Another topic of interest for W. Lance from the eighties of the last century was non-standard analysis. He (and not only he) was impressed by the fact (discovered when proving the Bernstein-Robinson theorem on invariant spaces) that it is impossible to build the Jordan form of an arbitrary linear operator being in ordinary Mathematics. It is necessary to take non-standard eigenvalues and non-standard principal vectors. So W. Lance developed the analysis of functions of discrete argument changing it by infinitesimal steps. Then he (jointly with Taras Kudryk, Yury Yavorsky, and Oxana Karabyn) investigated infinitesimal perturbations (in strong operator topology) of difference operators. He also built the theory of nearstandardness on finite sets.

Naturally, there are mathematicians in Lviv outside the Lance school. Prof. Yury Chersky investigated convolution-type equations in distribution spaces. Aleksandr Pankov investigated differential-operator equations and variation inequalities. His main result on this topic concerns the existence and regularity of solutions. He also (with Aleksandr Botsenyuk) investigated a new class of systems of differential-operator equations of the thermo-elasticity type and the structure of complex one-dimensional local Lie algebras (with Andriy Panasyuk).

Anatoliy Plichko studied biorthogonal systems in nonseparable spaces, weakly compactly generated spaces, some aspects of regularization of Tikhonov noncorrect problems, and random functions taking values in a Banach space. In particular he proved the existence of a total biorthogonal system in a Banach space, and the existence of a probability measure in a space of infinite cotype which has no space of finite type as its injective support.

Oleg Lopushansky investigated the spectral theory of locally convex algebras and algebras of unbounded operators in Banach spaces. This theory is based on new notions of semiregular spectrum and semiregular maximal ideals of a commutative algebra. Using these notions the Riesz-Dunford functional calculus has been built. Applications are di- 
rected towards the analysis of linear and semilinear differential operators; in particular, conditions of solvability of boundary evolutional equations, properties of stability and smoothness of solutions are investigated.

Andriy Zagorodnyuk works on polynomials and analytic functions on Banach spaces. In particular, Zagorodnyuk and Plichko proved that if $P$ is a discontinuous polynomial on a Banach space $X$, then there exists a sequence $\left\{x_{n}\right\}$ in $X$ such that $P\left(x+x_{n}\right)$ is unbounded for every $x$. This is an affirmative answer to Problem 56 by Mazur and Orlicz in "The Scottish Book". As consequences, some analogues were established for the Closed Graph and Open Mapping Theorems for polynomials on Banach spaces. Moreover, Zagorodnyuk (with Alencar, Aron and Galindo) described structures of sets of maximal ideals in various algebras of analytic functions with domains in Banach spaces.

\section{References}

[1] S. Albeverio, R. Hryniv, and Ya. Mykytyuk, Inverse spectral problems for Sturm-Liouville operators in impedance form, J. Funct. Anal. 222 (2005), no. 1, 143-177.

[2] R. Alencar, R. Aron, P. Galindo, and A. Zagorodnyuk, Algebra of symmetric holomorphic functions on $l_{p}$, Bull. Lond. Math. Soc. 35 (2003), 55-64.

[3] E. V. Cheremnykh, On a spectral expansion of some perturbed differential operator (in Russian), Ukrain. Mat. J. 41 (1989), no. 8, 1118-1183.

[4] E. V. Cheremnykh, Non-selfadjoint Friedrichs' model and Weyl's function (in Ukrainian), Dokl. Akad. Nauk Ukraine 8 (2001), 22-29.

[5] E. V. Cheremnykh, On normal eigenvalue embedded in continuous spectrum, Meth. Funct. Anal. and Topology 1 (2001), 1-16.

[6] R. O. Hryniv and Ya. V. Mykytyuk, $1 D$ Schrödinger operators with singular periodic potentials, Meth. Funct. Anal. and Topology 7 (2001), no. 4, 31-42.

[7] R. O. Hryniv and Ya. V. Mykytyuk, Inverse spectral problems for Sturm-Liouville operators with singular potentials, Inverse Problems 19 (2003), 665-684.

[8] R. O. Hryniv and Ya. V. Mykytyuk, Half-inverse spectral problems for Sturm-Liouville operators with singular potentials, Inverse Problems 20 (2004), no. 5, 1423-1444.

[9] W. E. Lance, Unbounded operators commuting with resolution of identity (in Russian), Ukrain. Mat. J. 15 (1963), no. 4, 376-384.

[10] W. E. Lance, A generalization of the concept of spectral measure (in Russian), Mat. Sb. 61 (103), (1963), 80-120.

[11] W. E. Lance, On a differential operator with spectral singularities (in Russian). I, Mat. Sb. 64 (106), (1964), 521-561; II, Mat. Sb. 65 (107), (1964), 47-103.

[12] W. E. Lance, Supplement I, in: Linear Differential Operators (in Russian), ed. M. A. Naimark, second edition, revised and augmented, Izdat. "Nauka", 1969, 443-498.

[13] W. E. Lance, Completely regular perturbation of a continuous spectrum (in Russian). I, Mat. Sb. 82 (124), (1970), 126-156; II, Mat. Sb. 84 (126), (1971), 141-158.

[14] W. E. Lance, Shadow of measure, Rend. Accad. Naz. Sci. XL Mem. Appl. 5 (1999), no. 23, $117-123$.

[15] W. E. Lance and G. I. Chuiko, Operator measure (in Russian), Dokl. Akad. Nauk Ukrain. SSR, Ser. A 7 (1981), 7-9.

[16] W. E. Lance and G. I. Chuiko, Rings of projectors and operator-valued measures, in: Spectral Theory and Its Applications, Theta, Bucharest 2003, pp. 151-158. 
[17] W. E. Lance and M. M. Fedyk, Properties of operators generated by sesquilinear forms (in Ukrainian), Dokl. Akad. Nauk Ukrain. SSR 2 (1987), 26-29.

[18] W. E. Lance and T. S. Kudryk, Introduction to Nonstandard Analysis, Math. Stud. Monogr. Ser. vol. 3, VNTL Publishers, Lviv 1997.

[19] W. Lance, A. Plichko, and O. Storozh, On investigations of Lvov mathematicians on functional analysis after 1945 (in Russian), Izdat. Lviv. Univers. 1992.

[20] W. E. Lance and O. G. Storozh, Methods of Theory of Unbounded Operators (in Russian), "Nauk. Dumka", Kiev 1983.

[21] O. V. Lopushansky, One-parameter semigroup in locally convex algebra (in Russian), Ukrain. Mat. J. 2 (1991), 23-27.

[22] O. V. Lopushansky, Bounded solutions of problem in locally convex algebras (in Russian), Dif. Uravn. 2 (1991), 357-359.

[23] Ya. V. Mykytyuk, Differential operators on the axis with a boundary condition at zero (in Russian), Ukrain. Mat. J. 34 (1982), no. 2, 119-222.

[24] Ya. V. Mykytyuk, Spectral theory of some class of non-selfadjoint operators (in Russian), Dokl. Akad. Nauk SSSR 252 (1990), no. 1, 40-43.

[25] Ya. V. Mykytyuk, On singular spectrum of non-selfadjoint operators (in Russian), Dokl. Akad. Nauk SSSR 303 (1988), no. 1, 33-36.

[26] Ya. V. Mykytyuk, Factorization of Fredholm operators (in Ukrainian), Mat. Stud. 20 (2003), no. 2, 185-199.

[27] O. Ya. Mylyo and O. G. Storozh, On general form of maximal accretive extension of positive operator (in Ukrainian), Dokl. Akad. Nauk Ukrain. SSR 6 (1991), 16-22.

[28] A. A. Pankov, Bounded and Periodic Solutions of Non-linear Differential-Operator Equations (in Russian), "Nauk. Dumka", Kiev 1985.

[29] A. M. Plichko, On bases and complements in non-separable Banach spaces (in Russian), Sib. Mat. J., I, 25 (1984), no. 4, 155-162; II, 27 (1986), no. 2, 149-153.

[30] A. Plichko and A. Zagorodnyuk, Isotropic mappings and automatic continuity of polynomial analytic and convex operators, in: General Topology in Banach Spaces, ed. T. Banakh, Nova Sci. Publ., Huntington, New York 2001, pp. 1-13.

[31] O. G. Storozh, Description of some class extensions of positive operators (in Ukrainian), Dokl. Akad. Nauk Ukrain. SSR 10 (1987), 14-16.

[32] O. G. Storozh, Accretive operators related to the positive one (in Russian), Ukrain. Mat. J. 41 (1989), no. 6, 789-794.

[33] O. G. Storozh and H. M. Pipa, On some perturbations changing the domain of proper extension of positively definite operator, Meth. of Funct. Anal. and Topology 11 (2005), no. 3, 257-269.

[34] I. P. Syroid, Sufficient conditions for spectral singularities of non-selfadjoint Schrödinger operator in potential terms (in Russian), Sib. Mat. J. 22 (1981), no. 1, 151-157.

[35] I. P. Syroid, Complex solutions of general Korteweg-de Vries equation: method of inverse problem (in Russian), Ukrain, Mat. J. 2 (1990), 223-230.

[36] I. P. Syroid, Spectral analysis over functional manifolds: Schrödinger analysis (in Ukrainian), Dop. Akad. Nauk of Ukraine 11 (1999), 43-48.

[37] A. V. Zagorodnyuk, On two statements of "The Scottish Book" concerning the ring of bounded polynomial functionals on Banach spaces (in Ukrainian), Ukrain. Mat. J. 48 (1996), no. 10, 1507-1516. 